\title{
Split hierarchical variational inequality problems and fixed point problems for nonexpansive mappings
}

\author{
Qamrul Hasan Ansari' , Aisha Rehan' and Ching-Feng Wen ${ }^{2,3 *}$
}

\author{
"Correspondence: \\ cfwen@kmu.edu.tw \\ ${ }^{2}$ Center for Fundamental Science, \\ Kaohsiung Medical University, \\ Kaohsiung, 807, Taiwan, ROC \\ ${ }^{3}$ Center for Nonlinear Analysis and \\ Optimization, Kaohsiung Medical \\ University, Kaohsiung, 807, Taiwan, \\ ROC \\ Full list of author information is \\ available at the end of the article
}

\begin{abstract}
The present paper deals with the common solution method for finding a fixed point of a nonexpansive mapping and a solution of split hierarchical Minty variational inequality problems. We discuss the weak convergence of the sequences generated by the proposed method to a common solution of a fixed point problem and a split hierarchical Minty variational inequality problem. An example is presented to illustrate the proposed algorithm and result.
\end{abstract}

MSC: 49J40; 49J52; 47J20

Keywords: split hierarchical variational inequality problems; fixed point problems; iterative method; nonexpansive mappings; convergence analysis

\section{Introduction}

Since its origin in 1994 by Censor and Elfving [1], the split feasibility problem (SFP) has been rapidly investigated and studied because of its applications in different areas such as signal processing, phase retrievals, image reconstruction, intensity-modulated radiation therapy, etc. (see, for example, [2-8] and the references therein). Recently, Censor and Segal [9] introduced a split common fixed point problem (SCFPP) which is to find a common element of a family of operators in one space such that its image under a linear transformation is a common fixed point of another family of operators in the image space. The SCFPP generalizes convex feasibility problem (CFP), split feasibility problem (SFP) and multiple sets split feasibility problem (MSSFP). They developed a parallel algorithm for solving SCFPP for the class of directed operators in the setting of finite-dimension spaces. Further, Cui et al. [10] proposed a damped projection method for SCFPP and studied its convergence result. Moudafi [11] further proposed and analyzed an iterative scheme for solving SCFPP for the class of demicontractive operators in the setting of Hilbert spaces. He studied the weak convergence of the sequence generated by the proposed algorithm to a solution of SCFPP. Subsequently, Cui and Wang [12] suggested a new algorithm that does not require any prior information of the operator norm to find a solution of SCFPP. They studied the weak convergence of the proposed algorithm. In 2011, Moudafi [13] considered the relaxed algorithm for computing the approximate solution of SCFPP for quasinonexpansive operators and studied the weak convergence of the sequence generated by the suggested algorithm. Very recently, SCFPP was considered by Kraikaew and Saejung

(c) 2015 Ansari et al. This article is distributed under the terms of the Creative Commons Attribution 4.0 International License (http://creativecommons.org/licenses/by/4.0/), which permits unrestricted use, distribution, and reproduction in any medium, provided you give appropriate credit to the original author(s) and the source, provide a link to the Creative Commons license, and indicate if changes were made. 
[14] for quasi-nonexpansive and strongly quasi-nonexpansive operators. They proposed an algorithm and showed that their algorithm converges strongly to a solution of SCFPP. They also considered split variational inequality problem [15], split common null point problem and Moudafi's split feasibility problem, and derived the algorithm for these problems from the main algorithm for SCFPP. Also, the strong convergence of these algorithms is derived from the main convergence result. Very recently, Ansari et al. [16] introduced the split hierarchical variational inequality problem (SHVIP). A variational inequality problem in which the underlying set is a set of fixed points of a nonlinear operator is called hierarchical variational inequality problem. For further details on hierarchical variational inequality problems, we refer to [17] and the references therein. More precisely, they considered the following split hierarchical Minty variational inequality problem (SHMVIP) which requires to find a solution of a hierarchical Minty variational inequality problem (HMVIP) such that its image under a nonlinear operator is a solution of another HMVIP.

Let $H_{1}$ and $H_{2}$ be real Hilbert spaces, $f, T: H_{1} \rightarrow H_{1}$ be operators such that $\operatorname{Fix}(T) \neq \emptyset$, and $h, S: H_{2} \rightarrow H_{2}$ be operators with $\operatorname{Fix}(S) \neq \emptyset$, where $\operatorname{Fix}(T)$ and $\operatorname{Fix}(S)$ are denoted by the set of fixed points of $T$ and $S$, respectively. Let $A: H_{1} \rightarrow H_{2}$ be an operator with $R(A) \cap \operatorname{Fix}(S) \neq \emptyset$, where $R(A)$ denotes the range of $A$. The split hierarchical variational inequality problem (SHVIP) is to find $x^{*} \in \operatorname{Fix}(T)$ such that

$$
\left\langle f\left(x^{*}\right), x-x^{*}\right\rangle \geq 0 \quad \text { for all } x \in \operatorname{Fix}(T)
$$

such that $A x^{*} \in \operatorname{Fix}(S)$ and it satisfies

$$
\left\langle h\left(A x^{*}\right), y-A x^{*}\right\rangle \geq 0 \quad \text { for all } y \in \operatorname{Fix}(S) .
$$

The solution set of the SHVIP is denoted by $\Omega$.

Another problem which is closely related to (SHVIP) is the following split hierarchical Minty variational inequality problem (SHMVIP): Find $x^{*} \in \operatorname{Fix}(T)$ such that

$$
\left\langle f(x), x-x^{*}\right\rangle \geq 0 \quad \text { for all } x \in \operatorname{Fix}(T),
$$

and such that $A x^{*} \in \operatorname{Fix}(S)$ satisfies

$$
\left\langle h(y), y-A x^{*}\right\rangle \geq 0 \quad \text { for all } y \in \operatorname{Fix}(S) \text {. }
$$

We denote by $\Gamma$ the set of solutions of SHMVIP, that is,

$$
\Gamma=\{x \text { solves }(3): A x \text { solves }(4)\}
$$

It can be easily seen by the Minty lemma [18], Lemma 1, that if $\operatorname{Fix}(T)$ and $\operatorname{Fix}(S)$ are nonempty closed convex and $f$ and $h$ are monotone and continuous, then SHVIP (1)-(2) and SHMVIP (3)-(4) are equivalent.

Ansari et al. [16] showed that several problems, namely split convex minimization problem, split variational inequality problem over the solution set of monotone variational inclusion problem, and split variational inequality problem over the solution set of equilibrium problem, are particular cases of SHVIP. They proposed an iterative scheme for solving SHVIP and studied the weak convergence of the sequence generated by the proposed algorithm. 
In this paper, we give a common solution method for finding a fixed point of a nonexpansive operator and a solution of split hierarchical variational inequality problems. The weak convergence of such algorithm is studied. We also present an example to illustrate the proposed algorithm and the convergence result.

\section{Preliminaries}

Let $H$ be a real Hilbert space whose inner product and norm are denoted by $\langle\cdot, \cdot\rangle$ and $\|\cdot\|$, respectively. Let $C$ be a nonempty closed convex subset of $H$. We denote by $x_{n} \rightarrow x$ (respectively, $x_{n} \rightarrow x$ ) the strong (respectively, weak) convergence of the sequence $\left\{x_{n}\right\}$ to $x$. Let $T: H \rightarrow H$ be an operator whose range is denoted by $R(T)$. The set of all fixed points of $T$ is denoted by $\operatorname{Fix}(T)$, that is, $\operatorname{Fix}(T)=\{x \in H: x=T x\}$.

Definition 2.1 An operator $T: H \rightarrow H$ is said to be:

(a) nonexpansive if $\|T x-T y\| \leq\|x-y\|$ for all $x, y \in H$;

(b) strongly nonexpansive $[19,20]$ if $T$ is nonexpansive and

$$
\lim _{n \rightarrow \infty}\left\|\left(x_{n}-y_{n}\right)-\left(T x_{n}-T y_{n}\right)\right\|=0
$$

whenever $\left\{x_{n}\right\}$ and $\left\{y_{n}\right\}$ are bounded sequences in $H$ and $\lim _{n \rightarrow \infty}\left(\left\|x_{n}-y_{n}\right\|-\left\|T x_{n}-T y_{n}\right\|\right)=0 ;$

(c) averaged nonexpansive if it can be written as

$$
T=(1-\alpha) I+\alpha S
$$

where $\alpha \in(0,1), I$ is the identity operator of $H$, and $S: H \rightarrow H$ is a nonexpansive mapping;

(d) firmly nonexpansive if $\|T x-T y\|^{2} \leq\langle x-y, T x-T y\rangle$ for all $x, y \in H$;

(e) cutter [20] if $\langle x-T x, z-T x\rangle \leq 0$ for all $x \in H$ and $z \in \operatorname{Fix}(T)$;

(f) monotone if $\langle x-y, T x-T y\rangle \geq 0$ for all $x, y \in H$;

(g) $\alpha$-inverse strongly monotone if there exists a constant $\alpha>0$ such that

$$
\langle T x-T y, x-y\rangle \geq \alpha\|T x-T y\|^{2} \quad \text { for all } x, y \in H
$$

Remark 2.1 Every strongly nonexpansive operator is nonexpansive, but a nonexpansive operator need not be strongly nonexpansive. Also, a nonexpansive cutter operator need not be strongly nonexpansive.

Example 2.1 Let $T:[-1,1] \rightarrow \mathbb{R}$ be defined by $T x=-x$ for all $x \in[-1,1]$. Then $T$ is nonexpansive but not strongly nonexpansive.

Indeed, let $x_{n}=1$ and $y_{n}=0$ for all $n$. Then $\left\{x_{n}\right\}$ and $\left\{y_{n}\right\}$ are bounded sequences. Also,

$$
\lim _{n \rightarrow \infty}\left|\left(x_{n}-y_{n}\right)-\left(T x_{n}-T y_{n}\right)\right|=\lim _{n \rightarrow \infty}|1+1|=2 \neq 0
$$

Thus, $T$ is not strongly nonexpansive.

Example 2.2 Let $H_{1}=H_{2}=\mathbb{R}^{2}$ with inner product and norm be given by $\langle x, y\rangle=x_{1} y_{1}+$ $x_{2} y_{2}$ and $\|x\|=\left|x_{1}\right|+\left|x_{2}\right|$, respectively, where $x=\left(x_{1}, x_{2}\right)$ and $y=\left(y_{1}, y_{2}\right)$. Let $C=\left\{x \in \mathbb{R}^{2}\right.$ : 
$\|x\| \leq \sqrt{2}$ \} be a nonempty closed subspace of $\mathbb{R}^{2}$. Let $T: C \rightarrow C$ be defined by

$$
T(x, y)=\left(\frac{2}{3} x+\frac{1}{3} y, \frac{1}{3} x+\frac{2}{3} y\right) \quad \text { for all }(x, y) \in C .
$$

Then $T$ is a nonexpansive cutter operator, but $T$ is not strongly nonexpansive.

Indeed, let $\left\{x_{n}\right\}=(1,0)$ and $\left\{y_{n}\right\}=(0,0)$ for all $n$. Then $\left\{x_{n}\right\}$ and $\left\{y_{n}\right\}$ are two bounded sequences of $C$, and $T x_{n}=\left(\frac{2}{3}, \frac{1}{3}\right)$ and $T y_{n}=(0,0)$. Note that

$$
\lim _{n \rightarrow \infty}\left(\left\|x_{n}-y_{n}\right\|-\left\|T x_{n}-T y_{n}\right\|\right)=\lim _{n \rightarrow \infty}\left(\|(1,0)\|-\left\|\left(\frac{2}{3}, \frac{1}{3}\right)\right\|\right)=\lim _{n \rightarrow \infty}(1-1)=0 .
$$

But

$$
\lim _{n \rightarrow \infty}\left\|\left(x_{n}-y_{n}\right)-\left(T x_{n}-T y_{n}\right)\right\|=\lim _{n \rightarrow \infty}\left\|(1,0)-\left(\frac{2}{3}, \frac{1}{3}\right)\right\|=\lim _{n \rightarrow \infty}\left\|\frac{1}{3}, \frac{-1}{3}\right\|=\frac{2}{3} \neq 0 .
$$

Thus, $T$ is not strongly nonexpansive.

In order to show that $T$ is a nonexpansive cutter operator, we first prove that it is nonexpansive. Let $x=\left(x_{1}, x_{2}\right), y=\left(y_{1}, y_{2}\right) \in C$. Then

$$
\begin{aligned}
\|T x-T y\| & =\left\|\left(\frac{2}{3} x_{1}+\frac{1}{3} x_{2}, \frac{1}{3} x_{1}+\frac{2}{3} x_{2}\right)-\left(\frac{2}{3} y_{1}+\frac{1}{3} y_{2}, \frac{1}{3} y_{1}+\frac{2}{3} y_{2}\right)\right\| \\
& =\left\|\frac{2}{3}\left(x_{1}-y_{1}\right)+\frac{1}{3}\left(x_{2}-y_{2}\right), \frac{1}{3}\left(x_{1}-y_{1}\right)+\frac{2}{3}\left(x_{2}-y_{2}\right)\right\| \\
& =\left|\frac{2}{3}\left(x_{1}-y_{1}\right)+\frac{1}{3}\left(x_{2}-y_{2}\right)\right|+\left|\frac{1}{3}\left(x_{1}-y_{1}\right)+\frac{2}{3}\left(x_{2}-y_{2}\right)\right| \\
& \leq \frac{2}{3}\left|x_{1}-y_{1}\right|+\frac{1}{3}\left|x_{2}-y_{2}\right|+\frac{1}{3}\left|x_{1}-y_{1}\right|+\frac{2}{3}\left|x_{2}-y_{2}\right| \\
& =\left|x_{1}-y_{1}\right|+\left|x_{2}-y_{2}\right|=\left\|\left(x_{1}-y_{1}\right),\left(x_{2}, y_{2}\right)\right\| \\
& =\left\|\left(x_{1}, x_{2}\right)-\left(y_{1}, y_{2}\right)\right\|=\|x-y\| .
\end{aligned}
$$

Thus, $T$ is nonexpansive.

We next show that $T$ is cutter. Note that $\operatorname{Fix}(T)=\{(x, y) \in C: x=y\}$. Let $x=(x, y) \in C$ and $(p, p) \in \operatorname{Fix}(T)$, we have

$$
\begin{aligned}
\langle x-T x, p-T x\rangle & =\left\langle(x, y)-\left(\frac{2}{3} x+\frac{1}{3} y, \frac{1}{3} x+\frac{2}{3} y\right),(p, p)-\left(\frac{2}{3} x+\frac{1}{3} y, \frac{1}{3} x+\frac{2}{3} y\right)\right\rangle \\
& =\left\langle\left(\frac{1}{3}(x-y), \frac{1}{3}(y-x)\right),\left(p-\frac{1}{3}(2 x+y), p-\frac{1}{3}(x+2 y)\right)\right\rangle \\
& =\frac{1}{3}(x-y)\left(p-\frac{1}{3}(2 x+y)\right)+\frac{1}{3}(y-x)\left(p-\frac{1}{3}(x+2 y)\right) \\
& =\frac{1}{9}(x-y)\{3 p-(2 x+y)-3 p+(x+2 y)\} \\
& =\frac{1}{9}(x-y)(y-x)=-\frac{1}{9}(x-y)^{2} \leq 0 .
\end{aligned}
$$

This shows that $T$ is cutter. 
The following lemma provides some fundamental properties of Hilbert spaces. These properties will be used in the sequel.

Lemma 2.1 Let $H$ be a real Hilbert space. Then, for all $x, y \in H$, we have

(a) $\|x-y\|^{2}=\|x\|^{2}-\|y\|^{2}-2\langle x-y, y\rangle$;

(b) $\|x-y\|^{2} \leq\|x\|^{2}+2\langle y, y-x\rangle$;

(c) $\|\lambda x+(1-\lambda) y\|^{2}=\lambda\|x\|^{2}+(1-\lambda)\|y\|^{2}-\lambda(1-\lambda)\|x-y\|^{2}$ for all $\lambda \in[0,1]$.

Lemma 2.2 ([21], Lemma 2, Demiclosedness principle) Let $C$ be a nonempty closed convex subset of a real Hilbert space $H$ and $T: C \rightarrow C$ be a nonexpansive operator with $\operatorname{Fix}(T) \neq \emptyset$. If the sequence $\left\{x_{n}\right\} \subseteq C$ converges weakly to $x$ and the sequence $\left\{(I-T) x_{n}\right\}$ converges strongly to $y$, then $(I-T) x=y$; in particular, if $y=0$, then $x \in \operatorname{Fix}(T)$.

Definition 2.2 Let $T: H \rightarrow 2^{H}$ be a set-valued operator with domain $D(T)=\{x \in H$ : $T(x) \neq \emptyset\}$, range $R(T)=\bigcup_{x \in D(T)} T(x)$ and the inverse of $T$ is $T^{-1}(y)=\{x \in H: y \in T(x)\}$. $T$ is said to be:

(a) monotone if

$$
\langle x-y, f-g\rangle \geq 0, \quad \text { whenever } f \in T x, g \in T y
$$

(b) maximal monotone if it is monotone and the graph

$$
G(T)=\{(x, f) \in H \times H: f \in T x\}
$$

of $T$ is not properly contained in the graph of any other monotone operator;

(c) $\alpha$-inverse strongly monotone if there exists a constant $\alpha>0$ such that

$$
\langle T x-T y, x-y\rangle \geq \alpha\|T x-T y\|^{2}, \quad \text { whenever } x, y \in D(T) .
$$

It is well known that when $T$ is maximal monotone, then for each $x \in H$ and $\lambda>0$, there is a unique $z \in H$ such that $x \in(I+\lambda T) z$. In this case, the operator $J_{\lambda}^{T}:=(I+\lambda T)^{-1}$ is called resolvent of $T$ with parameter $\lambda$. It is known that $J_{\lambda}^{T}$ is a single-valued and firmly nonexpansive mapping.

The following lemma will be used in our main result.

Lemma 2.3 ([22]) Let $\left\{a_{n}\right\}_{n=1}^{\infty}$ and $\left\{b_{n}\right\}_{n=1}^{\infty}$ be sequences of nonnegative real numbers such that

$$
a_{n+1} \leq a_{n}+b_{n} \text { for all } n \geq 1 \text {. }
$$

If $\sum_{n=1}^{\infty} b_{n}<\infty$, then the limit $\lim _{n \rightarrow \infty} a_{n}$ exists.

\section{Algorithms and convergence results}

Let $K: H_{1} \rightarrow H_{1}$ be a nonexpansive operator with Fix $(K) \cap \Gamma \neq \emptyset$. We propose the following algorithm to compute a common element of the set of fixed points of $K$ and the set of solutions of SHMVIP. 
Algorithm 3.1 Initialization: Choose $\left\{\alpha_{n}\right\}_{n=1}^{\infty},\left\{\beta_{n}\right\}_{n=1}^{\infty},\left\{\lambda_{n}\right\}_{n=1}^{\infty} \subset(0,1)$. Take arbitrary $x_{0} \in H_{1}$.

ITERATIVE STEP: For a given current $x_{n} \in H_{1}$, compute

$$
\begin{aligned}
& z_{n}=x_{n}-\gamma A^{*}\left(I-S\left(I-\beta_{n} h\right)\right) A x_{n}, \\
& y_{n}=T\left(I-\alpha_{n} f\right) z_{n}, \\
& x_{n+1}=\lambda_{n} x_{n}+\left(1-\lambda_{n}\right) K y_{n},
\end{aligned}
$$

where $\gamma \in\left(0, \frac{2}{\|A\|^{2}}\right)$.

LAST STEP: Update $n:=n+1$.

When $K$ is the identity operator, Algorithm 3.1 reduces to the following algorithm.

Algorithm 3.2 Initialization: Choose $\left\{\alpha_{n}\right\}_{n=1}^{\infty},\left\{\beta_{n}\right\}_{n=1}^{\infty},\left\{\lambda_{n}\right\}_{n=1}^{\infty} \subset(0,1)$. Take arbitrary $x_{0} \in H_{1}$.

ITERATIVE STEP: For a given current $x_{n} \in H_{1}$, compute

$$
\begin{aligned}
& z_{n}=x_{n}-\gamma A^{*}\left(I-S\left(I-\beta_{n} h\right)\right) A x_{n}, \\
& y_{n}=T\left(I-\alpha_{n} f\right) z_{n}, \\
& x_{n+1}=\lambda_{n} x_{n}+\left(1-\lambda_{n}\right) y_{n},
\end{aligned}
$$

where $\gamma \in\left(0, \frac{2}{\|A\|^{2}}\right)$.

LAST STEP: Update $n:=n+1$.

Next we prove the weak convergence of the sequences generated by Algorithm 3.1.

Theorem 3.1 Let $f: H_{1} \rightarrow H_{1}$ be a monotone continuous mapping, $T: H_{1} \rightarrow H_{1}$ be a nonexpansive cutter operator such that $\operatorname{Fix}(T) \neq \emptyset, h: H_{2} \rightarrow H_{2}$ be a monotone continuous mapping and $S: H_{2} \rightarrow H_{2}$ be a strongly nonexpansive cutter operator such that $\operatorname{Fix}(S) \neq \emptyset$. Let $A: H_{1} \rightarrow H_{2}$ be a bounded linear operator with $R(A) \cap \operatorname{Fix}(S) \neq \emptyset$ and let $K: H_{1} \rightarrow H_{1}$ be a nonexpansive operator with $\operatorname{Fix}(K) \cap \Gamma \neq \emptyset$. Let $\left\{x_{n}\right\}$ and $\left\{y_{n}\right\}$ be the sequences generated by Algorithm 3.1 such that the following conditions hold:

(i) There exists a natural number $n_{\circ}$ such that

$$
\Gamma \subset \bigcap_{n=n_{\circ}}^{\infty}\left\{z \in H_{1}:\left\langle h\left(A x_{n}\right), S\left(I-\beta_{n} h\right) A x_{n}-A z\right\rangle \geq 0\right\}
$$

(ii) $\left\{f\left(z_{n}\right)\right\}_{n=1}^{\infty}$ is a bounded sequence;

(iii) $\sum_{n=0}^{\infty} \alpha_{n}<\infty$;

(iv) $\lim _{n \rightarrow \infty} \beta_{n}=0$;

(v) $\left\|x_{n+1}-x_{n}\right\|=o\left(\alpha_{n}\right)$ and $\alpha_{n}=o\left(\beta_{n}^{2}\right)$;

(vi) $\left\{h\left(A x_{n}\right)\right\}_{n=1}^{\infty}$ is a bounded sequence.

Then the sequences $\left\{x_{n}\right\}$ and $\left\{y_{n}\right\}$ converge weakly to an element $x^{*} \in \operatorname{Fix}(K) \cap \Gamma$. 
Proof Let $p \in \operatorname{Fix}(K) \cap \Gamma$. Then $T(p)=p, K(p)=p$ and $S(A p)=A p$. Consider

$$
\begin{aligned}
\left\|z_{n}-p\right\|^{2}= & \left\|x_{n}-\gamma A^{*}\left(I-S\left(I-\beta_{n} h\right)\right) A x_{n}-p\right\|^{2} \\
= & \left\|x_{n}-p\right\|^{2}+\gamma^{2}\left\|A^{*}\left(I-S\left(I-\beta_{n} h\right)\right) A x_{n}\right\|^{2} \\
& -2 \gamma\left\langle x_{n}-p, A^{*}\left(I-S\left(I-\beta_{n} h\right)\right) A x_{n}\right\rangle \\
\leq & \left\|x_{n}-p\right\|^{2}+\gamma^{2}\|A\|^{2}\left\|\left(I-S\left(I-\beta_{n} h\right)\right) A x_{n}\right\|^{2} \\
& -2 \gamma\left\langle x_{n}-p, A^{*}\left(I-S\left(I-\beta_{n} h\right)\right) A x_{n}\right\rangle \quad \text { for all } n \geq 1 .
\end{aligned}
$$

Since $S$ is a cutter operator, we have

$$
\begin{aligned}
\left\langle x_{n}-\right. & \left.p, A^{*}\left(S\left(I-\beta_{n} h\right)-I\right) A x_{n}\right\rangle \\
= & \left\langle A x_{n}-A p,\left(S\left(I-\beta_{n} h\right)-I\right) A x_{n}\right\rangle \\
= & \left\langle S\left(I-\beta_{n} h\right) A x_{n}-A p+A x_{n}-S\left(I-\beta_{n} h\right) A x_{n},\left(S\left(I-\beta_{n} h\right)-I\right) A x_{n}\right\rangle \\
= & \left\langle S\left(I-\beta_{n} h\right) A x_{n}-A p,\left(S\left(I-\beta_{n} h\right)-I\right) A x_{n}\right\rangle-\left\|\left(S\left(I-\beta_{n} h\right)-I\right) A x_{n}\right\|^{2} \\
= & \left\langle S\left(I-\beta_{n} h\right) A x_{n}-A p,\left(S\left(I-\beta_{n} h\right)-I\right) A x_{n}+\beta_{n} h A x_{n}-\beta_{n} h A x_{n}\right\rangle \\
& -\left\|\left(S\left(I-\beta_{n} h\right)-I\right) A x_{n}\right\|^{2} \\
= & \left\langle S\left(I-\beta_{n} h\right) A x_{n}-A p, S\left(I-\beta_{n} h\right) A x_{n}-\left(I-\beta_{n} h\right) A x_{n}\right\rangle \\
& -\beta_{n}\left\langle S\left(I-\beta_{n} h\right)\left(A x_{n}\right)-A p, h\left(A x_{n}\right)\right\rangle-\left\|\left(S\left(I-\beta_{n} h\right)-I\right) A x_{n}\right\|^{2} \\
\leq & -\left\|\left(S\left(I-\beta_{n} h\right)-I\right) A x_{n}\right\|^{2}-\beta_{n}\left\langle S\left(I-\beta_{n} h\right)\left(A x_{n}\right)-A p, h\left(A x_{n}\right)\right\rangle .
\end{aligned}
$$

Since $p \in \Gamma$, by condition (i), we have

$$
\left\langle S\left(I-\beta_{n} h\right)\left(A x_{n}\right)-A p, h\left(A x_{n}\right)\right\rangle \geq 0 .
$$

Since $\beta_{n} \in(0,1)$ for all $n \in \mathbb{N}$, we further have

$$
\beta_{n}\left\langle S\left(I-\beta_{n} h\right)\left(A x_{n}\right)-A p, h\left(A x_{n}\right)\right\rangle \geq 0 .
$$

Therefore,

$$
\left\langle x_{n}-p, A^{*}\left(S\left(I-\beta_{n} h\right)-I\right) A x_{n}\right\rangle \leq-\left\|\left(S\left(I-\beta_{n} h\right)-I\right) A x_{n}\right\|^{2} .
$$

Thus, (7) becomes

$$
\begin{aligned}
\left\|z_{n}-p\right\|^{2} \leq & \left\|x_{n}-p\right\|^{2}+\gamma^{2}\|A\|^{2}\left\|\left(I-S\left(I-\beta_{n} h\right)\right) A x_{n}\right\|^{2} \\
& -2 \gamma\left\|\left(S\left(I-\beta_{n} h\right)-I\right) A x_{n}\right\|^{2} \\
= & \left\|x_{n}-p\right\|^{2}-\gamma\left(2-\gamma\|A\|^{2}\right)\left\|\left(S\left(I-\beta_{n} h\right)-I\right) A x_{n}\right\|^{2} \quad \text { for all } n \geq 1 .
\end{aligned}
$$

Since $\gamma \in\left(0, \frac{2}{\|A\|^{2}}\right)$, we observe that $\gamma\left(2-\gamma\|A\|^{2}\right)>0$, and hence

$$
\left\|z_{n}-p\right\| \leq\left\|x_{n}-p\right\| \quad \text { for all } n \geq 1 \text {. }
$$


Let $M:=\sup \left\{\left\|f\left(z_{n}\right)\right\|: n \geq 1\right\}$. Then, for all $n \geq 1$, we have

$$
\begin{aligned}
\left\|y_{n}-p\right\| & =\left\|T\left(z_{n}-\alpha_{n} f\left(z_{n}\right)\right)-T(p)\right\| \\
\leq & \left\|z_{n}-p\right\|+\alpha_{n}\left\|f\left(z_{n}\right)\right\| \\
\leq & \left\|z_{n}-p\right\|+\alpha_{n} M \\
\leq & \left\|x_{n}-p\right\|+\alpha_{n} M, \\
\left\|x_{n+1}-p\right\| & =\left\|\lambda_{n} x_{n}+\left(1-\lambda_{n}\right) K y_{n}-p\right\| \\
& =\left\|\lambda_{n}\left(x_{n}-p\right)+\left(1-\lambda_{n}\right)\left(K y_{n}-p\right)\right\| \\
& \leq \lambda_{n}\left\|x_{n}-p\right\|+\left(1-\lambda_{n}\right)\left\|y_{n}-p\right\| \\
& \leq \lambda_{n}\left\|x_{n}-p\right\|+\left(1-\lambda_{n}\right)\left\|x_{n}-p\right\|+\left(1-\lambda_{n}\right) \alpha_{n} M \\
& \leq\left\|x_{n}-p\right\|+\left(1-\lambda_{n}\right) \alpha_{n} M .
\end{aligned}
$$

Since $\sum \alpha_{n}<\infty$ and $0<\left(1-\lambda_{n}\right)<1$, we have $\sum_{n=1}^{\infty}\left(1-\lambda_{n}\right) \alpha_{n}<\infty$. Thus, by Lemma 2.3, the limit $\lim _{n \rightarrow \infty}\left\|x_{n}-p\right\|$ exists. Also, from (9)-(10), the limits $\lim _{n \rightarrow \infty}\left\|z_{n}-p\right\|$ and $\lim _{n \rightarrow \infty}\left\|y_{n}-p\right\|$ exist. This implies that $\left\{x_{n}\right\},\left\{y_{n}\right\}$ and $\left\{z_{n}\right\}$ are bounded sequences. Now, consider

$$
\begin{aligned}
\left\|y_{n}-p\right\|^{2} & =\left\|T\left(I-\alpha_{n} f\right)\left(z_{n}\right)-T(p)\right\|^{2} \\
& \leq\left\|\left(z_{n}-p\right)-\alpha_{n} f\left(z_{n}\right)\right\|^{2} \\
& \leq\left\|z_{n}-p\right\|^{2}+\alpha_{n}^{2}\left\|f\left(z_{n}\right)\right\|^{2}
\end{aligned}
$$

From (9), (11) and by Lemma 2.1(c), we have

$$
\begin{aligned}
\left\|x_{n+1}-p\right\|^{2}= & \left\|\lambda_{n} x_{n}+\left(1-\lambda_{n}\right) K y_{n}-p\right\|^{2} \\
= & \left\|\lambda_{n}\left(x_{n}-p\right)+\left(1-\lambda_{n}\right)\left(K y_{n}-p\right)\right\|^{2} \\
= & \lambda_{n}\left\|x_{n}-p\right\|^{2}+\left(1-\lambda_{n}\right)\left\|y_{n}-p\right\|^{2}-\lambda_{n}\left(1-\lambda_{n}\right)\left\|K y_{n}-x_{n}\right\|^{2} \\
\leq & \lambda_{n}\left\|x_{n}-p\right\|^{2}+\left(1-\lambda_{n}\right)\left\{\left\|z_{n}-p\right\|^{2}+\alpha_{n}^{2}\left\|f\left(z_{n}\right)\right\|^{2}\right\} \\
& -\lambda_{n}\left(1-\lambda_{n}\right)\left\|K y_{n}-x_{n}\right\|^{2} \\
\leq & \lambda_{n}\left\|x_{n}-p\right\|^{2}+\left(1-\lambda_{n}\right)\left\|x_{n}-p\right\|^{2} \\
& -\left(1-\lambda_{n}\right) \gamma\left(2-\gamma\|A\|^{2}\right)\left\|\left(S\left(I-\beta_{n} h\right)-I\right) A x_{n}\right\|^{2} \\
& +\left(1-\lambda_{n}\right) \alpha_{n}^{2}\left\|f\left(z_{n}\right)\right\|^{2}-\lambda_{n}\left(1-\lambda_{n}\right)\left\|K y_{n}-x_{n}\right\|^{2} \\
= & \left\|x_{n}-p\right\|-\left(1-\lambda_{n}\right) \gamma\left(2-\gamma\|A\|^{2}\right)\left\|\left(S\left(I-\beta_{n} h\right)-I\right) A x_{n}\right\|^{2} \\
& +\left(1-\lambda_{n}\right) \alpha_{n}^{2}\left\|f\left(z_{n}\right)\right\|^{2}-\lambda_{n}\left(1-\lambda_{n}\right)\left\|K y_{n}-x_{n}\right\|^{2},
\end{aligned}
$$

which is equivalent to

$$
\begin{aligned}
& \left(1-\lambda_{n}\right) \gamma\left(2-\gamma\|A\|^{2}\right)\left\|\left(S\left(I-\beta_{n} h\right)-I\right) A x_{n}\right\|^{2}+\lambda_{n}\left(1-\lambda_{n}\right)\left\|K y_{n}-x_{n}\right\|^{2} \\
& \quad \leq\left\|x_{n}-p\right\|^{2}-\left\|x_{n+1}-p\right\|^{2}+\left(1-\lambda_{n}\right) \alpha_{n}^{2}\left\|f\left(z_{n}\right)\right\|^{2} .
\end{aligned}
$$


From the existence of the limit $\lim _{n \rightarrow \infty}\left\|x_{n}-p\right\|$ and the facts that $\alpha_{n} \rightarrow 0,\left\|f\left(z_{n}\right)\right\|$ is bounded, $0<\lambda_{n}<1$ and $\gamma \in\left(0, \frac{2}{\|A\|^{2}}\right)$, it follows that

$$
\lim _{n \rightarrow \infty}\left\|\left(S\left(I-\beta_{n} h\right)-I\right) A x_{n}\right\|=0,
$$

and

$$
\lim _{n \rightarrow \infty}\left\|K y_{n}-x_{n}\right\|=0 .
$$

From (5), we have

$$
\lim _{n \rightarrow \infty}\left\|x_{n+1}-x_{n}\right\| \leq \lim _{n \rightarrow \infty}\left\|K y_{n}-x_{n}\right\|=0 .
$$

Since $T$ is a cutter operator, we have

$$
\begin{aligned}
\langle p- & \left.y_{n}, z_{n}-y_{n}\right\rangle \\
= & \left\langle y_{n}-p, y_{n}-z_{n}\right\rangle \\
= & \left\langle T\left(I-\alpha_{n} f\right) z_{n}-p, T\left(I-\alpha_{n} f\right) z_{n}-\left(I-\alpha_{n} f\right) z_{n}+\left(I-\alpha_{n} f\right) z_{n}-z_{n}\right\rangle \\
= & \left\langle T\left(I-\alpha_{n} f\right) z_{n}-p, T\left(I-\alpha_{n} f\right) z_{n}-\left(I-\alpha_{n} f\right) z_{n}\right\rangle \\
& +\left\langle T\left(I-\alpha_{n} f\right) z_{n}-p,-\alpha_{n} f z_{n}\right\rangle \\
\leq & \left\langle T\left(I-\alpha_{n} f\right) z_{n}-p, T\left(I-\alpha_{n} f\right) z_{n}-\left(I-\alpha_{n} f\right) z_{n}\right\rangle \\
& +\alpha_{n}\left\|T\left(I-\alpha_{n} f\right) z_{n}-p\right\|\left\|f z_{n}\right\|,
\end{aligned}
$$

and

$$
\left\langle T\left(I-\alpha_{n} f\right) z_{n}-p, T\left(I-\alpha_{n} f\right) z_{n}-\left(I-\alpha_{n} f\right) z_{n}\right\rangle \leq 0
$$

This implies that

$$
\left\langle p-y_{n}, z_{n}-y_{n}\right\rangle \leq \alpha_{n}\left\|T\left(I-\alpha_{n} f\right) z_{n}-p\right\|\left\|f z_{n}\right\| .
$$

From (9), (17) and by Lemma 2.1(a), we have

$$
\begin{aligned}
\left\|y_{n}-p\right\|^{2}= & \left\|z_{n}-p\right\|^{2}-\left\|z_{n}-y_{n}\right\|^{2}-2\left\langle y_{n}-p, z_{n}-y_{n}\right\rangle \\
\leq & \left\|x_{n}-p\right\|^{2}-\left\|x_{n}-\gamma A^{*}\left(I-S\left(I-\beta_{n} h\right)\right) A x_{n}-y_{n}\right\|^{2} \\
& -2\left\langle y_{n}-p, z_{n}-y_{n}\right\rangle \\
= & \left\|x_{n}-p\right\|^{2}-\left\|x_{n}-y_{n}\right\|^{2}-\gamma^{2}\|A\|^{2}\left\|\left(I-S\left(I-\beta_{n} h\right)\right) A x_{n}\right\|^{2} \\
& +2 \gamma\left\langle x_{n}-y_{n}, A^{*}\left(I-S\left(I-\beta_{n} h\right) A x_{n}\right)\right\rangle+2\left\langle p-y_{n}, z_{n}-y_{n}\right\rangle \\
\leq & \left\|x_{n}-p\right\|^{2}-\left\|x_{n}-y_{n}\right\|^{2}-\gamma^{2}\|A\|^{2}\left\|\left(I-S\left(I-\beta_{n} h\right)\right) A x_{n}\right\|^{2} \\
& +2 \alpha_{n}\left\|T\left(I-\alpha_{n} f\right) z_{n}-p\right\|\left\|z_{n}\right\| \\
& +2 \gamma\left\|x_{n}-y_{n}\right\|\|A\|\left\|\left(S\left(I-\beta_{n} h\right)-I\right) A x_{n}\right\| .
\end{aligned}
$$


Thus, from (12) and (18), we have

$$
\begin{aligned}
&\left\|x_{n+1}-p\right\|^{2} \\
&=\lambda_{n}\left\|x_{n}-p\right\|^{2}+\left(1-\lambda_{n}\right)\left\|y_{n}-p\right\|^{2}-\lambda_{n}\left(1-\lambda_{n}\right)\left\|K y_{n}-x_{n}\right\|^{2} \\
& \leq \lambda_{n}\left\|x_{n}-p\right\|^{2}+\left(1-\lambda_{n}\right)\left\|x_{n}-p\right\|^{2}-\left(1-\lambda_{n}\right) \gamma^{2}\|A\|^{2}\left\|\left(I-S\left(I-\beta_{n} h\right)\right) A x_{n}\right\|^{2} \\
&-\left(1-\lambda_{n}\right)\left\|x_{n}-y_{n}\right\|^{2}-\lambda_{n}\left(1-\lambda_{n}\right)\left\|K y_{n}-x_{n}\right\|^{2} \\
&+2\left(1-\lambda_{n}\right) \alpha_{n}\left\|T\left(I-\alpha_{n} f\right) z_{n}-p\right\|\left\|f\left(z_{n}\right)\right\| \\
&+2 \gamma\left(1-\lambda_{n}\right)\left\|x_{n}-y_{n}\right\|\|A\|\left\|\left(S\left(I-\beta_{n} h\right)-I\right) A x_{n}\right\| \\
& \leq\left\|x_{n}-p\right\|^{2}-\left(1-\lambda_{n}\right)\left\|x_{n}-y_{n}\right\|^{2}-\left(1-\lambda_{n}\right) \gamma^{2}\|A\|^{2}\left\|\left(I-S\left(I-\beta_{n} h\right)\right) A x_{n}\right\|^{2} \\
&+2\left(1-\lambda_{n}\right) \alpha_{n}\left\|T\left(I-\alpha_{n} f\right) z_{n}-p\right\|\left\|f\left(z_{n}\right)\right\|-\lambda_{n}\left(1-\lambda_{n}\right)\left\|K y_{n}-x_{n}\right\|^{2} \\
&+2 \gamma\left(1-\lambda_{n}\right)\left\|x_{n}-y_{n}\right\|\|A\|\left\|\left(S\left(I-\beta_{n} h\right)-I\right) A x_{n}\right\|,
\end{aligned}
$$

which is equivalent to

$$
\begin{aligned}
\left(1-\lambda_{n}\right)\left\|x_{n}-y_{n}\right\|^{2} \leq & \left\|x_{n}-p\right\|^{2}-\left\|x_{n+1}-p\right\|^{2}-\lambda_{n}\left(1-\lambda_{n}\right)\left\|K y_{n}-x_{n}\right\|^{2} \\
& +2\left(1-\lambda_{n}\right) \alpha_{n}\left\|T\left(I-\alpha_{n} f\right) z_{n}-p\right\|\left\|f\left(z_{n}\right)\right\| \\
& +2 \gamma\left(1-\lambda_{n}\right)\left\|x_{n}-y_{n}\right\|\|A\|\left\|\left(S\left(I-\beta_{n} h\right)-I\right) A x_{n}\right\| .
\end{aligned}
$$

Taking limit as $n \rightarrow \infty$, and taking into account $\alpha_{n} \rightarrow 0,0<\lambda_{n}<1, \gamma \in\left(0, \frac{2}{\|A\|^{2}}\right)$ and from equations (14), (15) we have

$$
\begin{aligned}
\left\|x_{n}-y_{n}\right\| & \rightarrow 0 \quad \text { as } n \rightarrow \infty \\
\left\|y_{n}-K y_{n}\right\| & =\left\|y_{n}-x_{n}+x_{n}-K y_{n}\right\| \\
& \leq\left\|y_{n}-x_{n}\right\|+\left\|x_{n}-K y_{n}\right\| .
\end{aligned}
$$

From (15) and (19), we obtain

$$
\left\|y_{n}-K y_{n}\right\| \rightarrow 0 \quad \text { as } n \rightarrow \infty
$$

Since $\left\{x_{n}\right\}$ is a bounded sequence, there exists a convergent subsequence $\left\{x_{n_{i}}\right\}$ of $\left\{x_{n}\right\}$ that converges weakly to some $x^{*} \in H_{1}$. Since $\left\|x_{n}-y_{n}\right\| \rightarrow 0$, it is known that $y_{n_{i}} \rightarrow x^{*} \in H_{1}$. By the demiclosed principle, $y_{n_{i}} \rightarrow x^{*}$ and $\left\|y_{n_{i}}-K y_{n_{i}}\right\| \rightarrow 0$, we have

$$
K x^{*}=x^{*} \text {. }
$$

From (5), we obtain

$$
\left\|z_{n}-x_{n}\right\|=\gamma\|A\|\left\|\left(I-S\left(I-\beta_{n} h\right)\right) A x_{n}\right\| .
$$

By (14), we have

$$
\left\|z_{n}-x_{n}\right\| \rightarrow 0 \quad \text { as } n \rightarrow \infty,
$$


and

$$
\left\|z_{n}-y_{n}\right\| \leq\left\|x_{n}-y_{n}\right\|+\gamma\|A\|\left\|\left(I-S\left(I-\beta_{n} h\right)\right) A x_{n}\right\| .
$$

Equations (14) and (19) yield that

$$
\left\|z_{n}-y_{n}\right\| \rightarrow 0 \quad \text { as } n \rightarrow \infty \text {. }
$$

From the definition of $y_{n}$, we have

$$
\begin{aligned}
\left\|y_{n}-T z_{n}\right\| & =\left\|T\left(z_{n}-\alpha_{n} f\left(z_{n}\right)\right)-T z_{n}\right\| \\
& \leq\left\|z_{n}-\alpha_{n} f\left(z_{n}\right)-z_{n}\right\| \\
& \leq \alpha_{n}\left\|f\left(z_{n}\right)\right\| .
\end{aligned}
$$

This implies that

$$
\begin{aligned}
\lim _{n \rightarrow \infty}\left\|y_{n}-T z_{n}\right\|=0, & \\
\left\|y_{n}-T y_{n}\right\| & =\left\|y_{n}-T z_{n}+T z_{n}-T y_{n}\right\| \\
& \leq\left\|y_{n}-T z_{n}\right\|+\left\|z_{n}-y_{n}\right\| .
\end{aligned}
$$

From (22) and (23), we get

$$
\lim _{n \rightarrow \infty}\left\|y_{n}-T y_{n}\right\| \rightarrow 0
$$

Since $y_{n_{i}} \rightarrow x^{*}$ and $\left\|y_{n_{i}}-T y_{n_{i}}\right\| \rightarrow 0$, by the demiclosed principle, we obtain

$$
T x^{*}=x^{*} \text {. }
$$

Let $v_{n}:=A x_{n}-\beta_{n} h\left(A x_{n}\right)$ for all $n \geq 1$. We observe that

$$
\begin{aligned}
0 & \leq\left\|v_{n}-A p\right\|-\left\|S v_{n}-S A p\right\| \\
& =\left\|A x_{n}-\beta_{n} h\left(A x_{n}\right)-A p\right\|-\left\|S v_{n}-S A p\right\| \\
& =\left\|A x_{n}-S v_{n}+S v_{n}-\beta_{n} h\left(A x_{n}\right)-A p\right\|-\left\|S v_{n}-S A p\right\| \\
& \leq\left\|A x_{n}-S v_{n}\right\|+\left\|S v_{n}-S A p\right\|+\beta_{n}\left\|h\left(A x_{n}\right)\right\|-\left\|S v_{n}-S A p\right\| \\
& =\left\|A x_{n}-S\left(A x_{n}-\beta_{n}\left(h\left(A x_{n}\right)\right)\right)\right\|+\beta_{n}\left\|h\left(A x_{n}\right)\right\| \\
& =\left\|\left(S\left(I-\beta_{n} h\right)-I\right) A x_{n}\right\|+\beta_{n}\left\|h\left(A x_{n}\right)\right\| .
\end{aligned}
$$

From condition (iv) and (14), we have

$$
\lim _{n \rightarrow \infty}\left(\left\|v_{n}-A p\right\|-\left\|S v_{n}-S A p\right\|\right)=0
$$

The boundedness of $v_{n}$ and strong nonexpansiveness of $S$ imply that

$$
\lim _{n \rightarrow \infty}\left\|S v_{n}-v_{n}\right\|=0
$$


From the definition of $v_{n}$ and condition (iv), we get

$$
\lim _{n \rightarrow \infty}\left\|v_{n}-A x_{n}\right\|=0
$$

and

$$
\begin{aligned}
\left\|v_{n}-S A x_{n}\right\| & \leq\left\|v_{n}-S v_{n}\right\|+\left\|S v_{n}-S\left(A x_{n}\right)\right\| \\
& =\left\|v_{n}-S v_{n}\right\|+\left\|v_{n}-A x_{n}\right\| .
\end{aligned}
$$

From (24) and (25), we have

$$
\lim _{n \rightarrow \infty}\left\|v_{n}-S A x_{n}\right\|=0
$$

and thus

$$
\lim _{n \rightarrow \infty}\left\|A x_{n}-S A x_{n}\right\|=0 \text {. }
$$

Since $x_{n_{i}} \rightarrow x^{*} \in H_{1}$, we have $A x_{n_{i}} \rightarrow A x^{*} \in H_{2}$. From (26) and by the demiclosed principle, we obtain

$$
S A x^{*}=A x^{*} .
$$

Let $q_{n}:=z_{n}-\alpha_{n} f\left(z_{n}\right)$. By Lemma 2.1(b) and inequality (9), we have

$$
\begin{aligned}
\left\|y_{n}-p\right\|^{2} & =\left\|T\left(I-\alpha_{n} f\right) z_{n}-T p\right\|^{2} \\
& \leq\left\|z_{n}-p-\alpha_{n} f\left(z_{n}\right)\right\|^{2} \\
& \leq\left\|z_{n}-p\right\|^{2}+2\left\langle\alpha_{n} f\left(z_{n}\right), \alpha_{n} f\left(z_{n}\right)-z_{n}+p\right\rangle \\
& =\left\|z_{n}-p\right\|^{2}+2\left\langle\alpha_{n} f\left(z_{n}\right), p-z_{n}\right\rangle+2 \alpha_{n}^{2}\left\|f\left(z_{n}\right)\right\|^{2} \\
& \leq\left\|x_{n}-p\right\|^{2}+2 \alpha_{n}\left\langle f\left(z_{n}\right), p-z_{n}\right\rangle+2 \alpha_{n}^{2}\left\|f\left(z_{n}\right)\right\|^{2} .
\end{aligned}
$$

From the definition of $x_{n+1},(27)$ and using the monotonicity of $f$, we have

$$
\begin{aligned}
\left\|x_{n+1}-p\right\|^{2} \leq & \lambda_{n}\left\|x_{n}-p\right\|^{2}+\left(1-\lambda_{n}\right)\left\|y_{n}-p\right\|^{2} \\
\leq & \lambda_{n}\left\|x_{n}-p\right\|^{2}+\left(1-\lambda_{n}\right)\left\{\left\|x_{n}-p\right\|^{2}+2 \alpha_{n}\left\langle f\left(z_{n}\right), p-z_{n}\right\rangle\right. \\
& \left.+2 \alpha_{n}^{2}\left\|f\left(z_{n}\right)\right\|^{2}\right\} \\
\leq & \left\|x_{n}-p\right\|^{2}+2\left(1-\lambda_{n}\right) \alpha_{n}\left\langle f\left(z_{n}\right), p-z_{n}\right\rangle+2\left(1-\lambda_{n}\right) \alpha_{n}^{2}\left\|f\left(z_{n}\right)\right\|^{2} \\
= & \left\|x_{n}-p\right\|^{2}+2\left(1-\lambda_{n}\right) \alpha_{n}\left\langle f\left(z_{n}\right)-f(p)+f(p), p-z_{n}\right\rangle \\
& +2\left(1-\lambda_{n}\right) \alpha_{n}^{2}\left\|f\left(z_{n}\right)\right\|^{2} \\
= & \left\|x_{n}-p\right\|^{2}+2\left(1-\lambda_{n}\right) \alpha_{n}\left\langle f\left(z_{n}\right)-f(p), p-z_{n}\right\rangle \\
& +2\left(1-\lambda_{n}\right) \alpha_{n}\left\langle f(p), p-z_{n}\right\rangle+2\left(1-\lambda_{n}\right) \alpha_{n}^{2}\left\|f\left(z_{n}\right)\right\|^{2} \\
= & \left\|x_{n}-p\right\|^{2}-2\left(1-\lambda_{n}\right) \alpha_{n}\left\langle f(p)-f\left(z_{n}\right), p-z_{n}\right\rangle
\end{aligned}
$$




$$
\begin{aligned}
& +2\left(1-\lambda_{n}\right) \alpha_{n}\left\langle f(p), p-z_{n}\right\rangle+2\left(1-\lambda_{n}\right) \alpha_{n}^{2}\left\|f\left(z_{n}\right)\right\|^{2} \\
= & \left\|x_{n}-p\right\|^{2}+2\left(1-\lambda_{n}\right) \alpha_{n}\left\langle f(p), p-z_{n}\right\rangle+2\left(1-\lambda_{n}\right) \alpha_{n}^{2}\left\|f\left(z_{n}\right)\right\|^{2},
\end{aligned}
$$

which is equivalent to

$$
\begin{aligned}
2 & \left(1-\lambda_{n}\right)\left\{f(p), z_{n}-p\right\rangle \\
& \leq\left(\frac{\left\|x_{n}-p\right\|^{2}-\left\|x_{n+1}-p\right\|^{2}}{\alpha_{n}}\right)+2\left(1-\lambda_{n}\right) \alpha_{n}\left\|f\left(z_{n}\right)\right\|^{2} \\
& \leq\left\{\frac{\left(\left\|x_{n}-p\right\|+\left\|x_{n+1}-p\right\|\right)\left(\left\|x_{n}-p\right\|-\left\|x_{n+1}-p\right\|\right)}{\alpha_{n}}\right\}+2\left(1-\lambda_{n}\right) \alpha_{n}\left\|f\left(z_{n}\right)\right\|^{2} \\
& \leq M_{1}\left(\frac{\left\|x_{n}-p\right\|-\left\|x_{n+1}-p\right\|}{\alpha_{n}}\right)+2\left(1-\lambda_{n}\right) \alpha_{n}\left\|f\left(z_{n}\right)\right\|^{2} \\
& \leq M_{1}\left(\frac{\left\|x_{n}-x_{n+1}\right\|}{\alpha_{n}}\right)+2\left(1-\lambda_{n}\right) \alpha_{n}\left\|f\left(z_{n}\right)\right\|^{2},
\end{aligned}
$$

where $M_{1}=\sup \left\{\left\|x_{n}-p\right\|+\left\|x_{n+1}-p\right\|, n \geq 1\right\}<\infty$. Taking limit of both sides and taking into account that $0<\left(1-\lambda_{n}\right)<1, \alpha_{n} \rightarrow 0,\left\|x_{n}-x_{n+1}\right\|=o\left(\alpha_{n}\right)$ and $z_{n_{i}} \rightarrow x^{*}$, we have

$$
\left\langle f(p), x^{*}-p\right\rangle \leq 0
$$

and thus

$$
\left\langle f(p), x^{*}-p\right\rangle \leq 0 \quad \text { for all } p \in \operatorname{Fix}(T)
$$

that is, $x^{*} \in \operatorname{Fix}(T)$ solves (3). Since $\alpha_{n}=o\left(\beta_{n}^{2}\right)$, we may assume that $\alpha_{n} \leq \beta_{n}^{2}$ for all $n \geq 1$. From (13), for all $n \geq 1$, we have

$$
\begin{aligned}
(1 & \left.-\lambda_{n}\right) \gamma\left(2-\gamma\|A\|^{2}\right)\left\|\left(S\left(I-\beta_{n} h\right)-I\right) A x_{n}\right\|^{2} \\
& \leq\left\|x_{n}-p\right\|^{2}-\left\|x_{n+1}-p\right\|^{2}+\alpha_{n}^{2}\left\|f\left(z_{n}\right)\right\|^{2} \\
& \leq\left\{\left(\left\|x_{n}-p\right\|+\left\|x_{n+1}-p\right\|\right)\left(\left\|x_{n}-p\right\|-\left\|x_{n+1}-p\right\|\right)\right\}+\alpha_{n}^{2}\left\|f\left(z_{n}\right)\right\|^{2} \\
& \leq M_{2}\left\|x_{n}-x_{n+1}\right\|+\alpha_{n}^{2}\left\|f\left(z_{n}\right)\right\|^{2},
\end{aligned}
$$

where $M_{2}=\sup \left\{\left\|x_{n}-p\right\|+\left\|x_{n+1}-p\right\|: n \geq 1\right\}<\infty$. Therefore, for all $n \geq 1$, we have

$$
\begin{aligned}
\left(1-\lambda_{n}\right) \gamma\left(2-\gamma\|A\|^{2}\right) \frac{\left\|A x_{n}-S v_{n}\right\|^{2}}{\beta_{n}^{2}} & \leq \frac{\left\|x_{n}-x_{n+1}\right\|}{\beta_{n}^{2}} M_{2}+\frac{\alpha_{n}^{2}}{\beta_{n}^{2}}\left\|f\left(z_{n}\right)\right\|^{2} \\
& \leq \frac{\left\|x_{n}-x_{n+1}\right\|}{\alpha_{n}} M_{2}+\alpha_{n}\left\|f\left(z_{n}\right)\right\|^{2} .
\end{aligned}
$$

Subsequently, since $\alpha_{n} \rightarrow 0,\left\|x_{n+1}-x_{n}\right\|=o\left(\alpha_{n}\right), \gamma\left(2-\gamma\|A\|^{2}\right)>0$ and $0<\left(1-\lambda_{n}\right)<1$, we have

$$
\lim _{n \rightarrow \infty} \frac{\left\|A x_{n}-S v_{n}\right\|}{\beta_{n}}=0 .
$$


For all $n \geq 1$, by Lemma 2.1(b) and the monotonicity of $h$, we compute

$$
\begin{aligned}
&\left\|S v_{n}-S A p\right\|^{2} \\
& \leq\left\|v_{n}-A p\right\| \\
& \leq\left\|A x_{n}-\beta_{n} h\left(A x_{n}\right)-A p\right\|^{2} \\
& \leq\left\|A x_{n}-A p\right\|^{2}+2\left\langle\beta_{n} h\left(A x_{n}\right), \beta_{n} h\left(A x_{n}\right)-A x_{n}+A p\right\rangle \\
& \leq\left\|A x_{n}-A p\right\|^{2}+2 \beta_{n}\left\langle h\left(A x_{n}\right), A p-A x_{n}\right\rangle+2 \beta_{n}^{2}\left\|h\left(A x_{n}\right)\right\|^{2} \\
& \leq\left\|A x_{n}-A p\right\|^{2}-2 \beta_{n}\left\langle h(A p)-h\left(A x_{n}\right), A p-A x_{n}\right\rangle \\
&+2 \beta_{n}^{2}\left\|h\left(A x_{n}\right)\right\|^{2}+2 \beta_{n}\left\langle h(A p), A p-A x_{n}\right\rangle \\
& \leq\left\|A x_{n}-A p\right\|^{2}+2 \beta_{n}^{2}\left\|h\left(A x_{n}\right)\right\|^{2}+2 \beta_{n}\left\langle h(A p), A p-A x_{n}\right\rangle .
\end{aligned}
$$

This gives

$$
\begin{aligned}
& 2\left\langle h(A p), A x_{n}-A p\right\rangle \\
& \quad \leq\left\{\frac{\left\|A x_{n}-A p\right\|^{2}-\left\|S v_{n}-S A p\right\|^{2}}{\beta_{n}}\right\}+2 \beta_{n}\left\|h\left(A x_{n}\right)\right\|^{2} \\
& \quad \leq\left\{\frac{\left(\left\|A x_{n}-A p\right\|-\left\|S v_{n}-S A p\right\|\right)\left(\left\|A x_{n}-A p\right\|+\left\|S v_{n}-S A p\right\|\right)}{\beta_{n}}\right\}+2 \beta_{n}\left\|h\left(A x_{n}\right)\right\|^{2} \\
& \quad \leq\left(\frac{\left\|A x_{n}-S v_{n}\right\|}{\beta_{n}}\right) M_{4}+2 \beta_{n}\left\|h\left(A x_{n}\right)\right\|^{2},
\end{aligned}
$$

where $M_{4}:=\sup \left\{\left\|A x_{n}-A p\right\|+\left\|S v_{n}-S A p\right\|: n \geq 1\right\}<\infty$. From (29), condition (iv) and $A x_{n} \rightarrow A p$, we obtain

$$
\left\langle h(A p), A x^{*}-A p\right\rangle \leq 0 \quad \text { for all } A p \in \operatorname{Fix}(S)
$$

that is, $A x^{*}$ solves (4). Finally, it remains to show that $x_{n} \rightarrow x^{*}$. Note that, by the boundedness of $\left\{x_{n}\right\}$, it suffices to show that there is no subsequence $\left\{x_{n_{i}}\right\}$ of $\left\{x_{n}\right\}$ such that $x_{n_{i}} \rightarrow y^{*} \in H_{1}$ and $y^{*} \neq x^{*}$.

Indeed, if this is not true, then the well-known Opial theorem would imply

$$
\begin{aligned}
\lim _{n \rightarrow \infty}\left\|x_{n}-y^{*}\right\| & =\lim _{j \rightarrow \infty}\left\|x_{n_{j}}-x^{*}\right\|<\lim _{j \rightarrow \infty}\left\|x_{n_{j}}-y^{*}\right\| \\
& =\lim _{n \rightarrow \infty}\left\|x_{n}-y^{*}\right\|=\lim _{i \rightarrow \infty}\left\|x_{n_{i}}-y^{*}\right\| \\
< & \lim _{i \rightarrow \infty}\left\|x_{n_{i}}-x^{*}\right\|=\lim _{n \rightarrow \infty}\left\|x_{n}-y^{*}\right\|,
\end{aligned}
$$

which leads to a contradiction. Therefore, the sequence $\left\{x_{n}\right\}_{n=1}^{\infty}$ converges weakly to a solution $x^{*} \in \Gamma$. Thus, $\left\|x_{n}-y_{n}\right\| \rightarrow 0$ and $\left\|x_{n}-z_{n}\right\| \rightarrow 0$ imply that $y_{n} \rightarrow x^{*}$ and $z_{n} \rightarrow x^{*}$, respectively.

Remark 3.1 By taking $K \equiv I$, the identity operator in Theorem 3.1, we can derive a weak convergence result for Algorithm 3.2 in which the operator $T$ is nonexpansive cutter, but not strongly nonexpansive as in [16]. 
Now, we illustrate Algorithm 3.1 and Theorem 3.1 by the following example.

Example 3.1 Let $H_{1}, H_{2}, C$ and $T$ be the same as in Example 2.2. Let $S: C \rightarrow C$ be defined as

$$
S(x, y)=\left(\frac{1}{10} x+\frac{1}{10} y, \frac{1}{10} x+\frac{1}{10} y\right) \quad \text { for all }(x, y) \in C .
$$

Then $S$ is strongly nonexpansive cutter and firmly nonexpansive, and it has a fixed point $(0,0)$. Thus being firmly nonexpansive, $S$ is strongly nonexpansive. Also, every firmly nonexpansive operator with a fixed point is cutter (see $[16,20])$. Thus, $S$ is a strongly nonexpansive cutter operator.

Let $f, h: C \rightarrow C$ be operators defined by

$$
f(x, y)=\left(\frac{1}{3} x-\frac{1}{3} y,-\frac{1}{3} x+\frac{1}{3} y\right) \text { for all }(x, y) \in C,
$$

and

$$
h(x, y)=\left(\frac{1}{2} x-\frac{1}{2} y,-\frac{1}{2} x+\frac{1}{2} y\right) \quad \text { for all }(x, y) \in C .
$$

Then $f$ and $h$ are monotone.

Let $K: C \rightarrow C$ be defined by

$$
K(x, y)=\left(\frac{1}{5} x+\frac{1}{5} y, \frac{1}{5} x+\frac{1}{5} y\right) \quad \text { for all }(x, y) \in C .
$$

Then $K$ is nonexpansive.

Let $A: C \rightarrow C$ be defined by

$$
A(x, y)=\left(\frac{1}{2} x, \frac{1}{2} y\right) \quad \text { for all }(x, y) \in C .
$$

Then $A$ is a bounded linear operator and $\|A\|^{2}=\frac{1}{4}$.

Table 1 Convergence table of Example 3.1

\begin{tabular}{lcc}
\hline No. of iterations $(\boldsymbol{n})$ & $\boldsymbol{y}^{\boldsymbol{n}}$ & $\boldsymbol{x}^{\boldsymbol{n}}$ \\
\hline 1 & $(-0.2222,0.2222)$ & $(1,-1)$ \\
2 & $(1.0 \mathrm{e}-004)(-0.1528,0.1528)$ & $(1.0 \mathrm{e}-004)(0.5000,-0.5000)$ \\
3 & $(1.0 \mathrm{e}-008)(-0.1605,0.1605)$ & $(1.0 \mathrm{e}-008)(0.5000,-0.5000)$ \\
4 & $(1.0 \mathrm{e}-012)(-0.2447,0.2447)$ & $(1.0 \mathrm{e}-012)(0.7500,-0.7500)$ \\
5 & $(1.0 \mathrm{e}-016)(-0.4931,0.4931)$ & $(1.0 \mathrm{e}-015)(0.1500,-0.1500)$ \\
6 & $(1.0 \mathrm{e}-019)(-0.1238,0.1238)$ & $(1.0 \mathrm{e}-019)(0.3750,-0.3750)$ \\
7 & $(1.0 \mathrm{e}-023)(-0.3722,0.3722)$ & $(1.0 \mathrm{e}-022)(0.1125,-0.1125)$ \\
8 & $(1.0 \mathrm{e}-026)(-0.1305,0.1305)$ & $(1.0 \mathrm{e}-026)(0.3938,-0.3938)$ \\
9 & $(1.0 \mathrm{e}-030)(-0.5223,0.5223)$ & $(1.0 \mathrm{e}-029)(0.1575,-0.1575)$ \\
10 & $(1.0 \mathrm{e}-033)(-0.2352,0.2352)$ & $(1.0 \mathrm{e}-033)(0.7088,-0.7088)$ \\
11 & $(1.0 \mathrm{e}-036)(-0.1177,0.1177)$ & $(1.0 \mathrm{e}-036)(0.5544,-0.5544)$ \\
12 & $(0,0)$ & $(0,0)$ \\
13 & $(0,0)$ & $(0,0)$ \\
14 & $(0,0)$ & $(0,0)$ \\
15 & $(0,0)$ & $(0,0)$ \\
16 & $(0,0)$ & $(0,0)$ \\
\hline
\end{tabular}


Let $\alpha_{n}=\left(1 / 2 n^{2}, 1 / 2 n^{2}\right), \beta_{n}=(1 / n, 1 / n), \gamma \in(0,8)$ and $\lambda_{n} \in(0,1)$. Then the sequences $x_{n}$ and $y_{n}$ generated by Algorithm 3.1 with initial guess $x^{1}=(1,-1)$ converge to $(0,0)$ (see Table 1 ) which is a fixed point of $T$ and $K$, whereas $A(0,0)=(0,0)$ which is the fixed point of $S$, where $x^{1}=\left(x_{1}^{1}, x_{2}^{1}\right)$. Thus, $(0,0)$ is the required solution.

\section{Competing interests}

The authors declare that they have no competing interests.

\section{Authors' contributions}

All authors have equal contribution.

\section{Author details}

${ }^{1}$ Department of Mathematics, Aligarh Muslim University, Aligarh, India. ${ }^{2}$ Center for Fundamental Science, Kaohsiung Medical University, Kaohsiung, 807, Taiwan, ROC. ${ }^{3}$ Center for Nonlinear Analysis and Optimization, Kaohsiung Medical University, Kaohsiung, 807, Taiwan, ROC.

\section{Acknowledgements}

In this research, the last author was partially supported by the Grant MOST 104-2115-M-037-001.

\section{Received: 27 March 2015 Accepted: 23 August 2015 Published online: 17 September 2015}

\section{References}

1. Censor, Y, Elfving, T: A multiprojection algorithm using Bregman projections in a product space. Numer. Algorithms 8 , 221-239 (1994)

2. Ansari, QH, Rehan, A: Split feasibility and fixed point problems. In: Ansari, QH (ed.) Nonlinear Analysis: Approximation Theory, Optimization and Applications, pp. 281-322. Springer, New York (2014)

3. Byrne, C: Iterative oblique projection onto convex subsets and the split feasibility problem. Inverse Probl. 18, 441-453 (2002)

4. Byrne, C: A unified treatment of some iterative algorithms in signal processing and image reconstruction. Inverse Probl. 20, 103-120 (2004)

5. Censor, Y, Bortfeld, T, Martin, B, Trofimov, A: A unified approach for inversion problems in intensity-modulated radiation therapy. Phys. Med. Biol. 51, 2353-2365 (2006)

6. Ceng, L-C, Ansari, QH, Yao, J-C: An extragradient method for solving split feasibility and fixed point problems. Comput. Math. Appl. 64, 633-642 (2012)

7. Ceng, L-C, Ansari, QH, Yao, J-C: Mann type iterative methods for finding a common solution of split feasibility and fixed point problems. Positivity 16, 471-495 (2012)

8. Ceng, L-C, Wong, M-M, Yao, J-C: A hybrid extragradient-like approximation method with regularization for solving split feasibility and fixed point problems. J. Nonlinear Convex Anal. 14, 163-182 (2013)

9. Censor, Y, Segal, A: The split common fixed point problems for directed operators. J. Convex Anal. 16, 587-600 (2009)

10. Cui, H, Su, M, Wang, F: Damped projection method for split common fixed point problems. Fixed Point Theory Appl. 2013, Article ID 123 (2013)

11. Moudafi, A: The split common fixed-point problem for demicontractive mappings. Inverse Probl. 26, 1-6 (2010)

12. Cui, H, Wang, F: Iterative method for the split common fixed point problem in Hilbert spaces. Fixed Point Theory Appl. 2014, Article ID 78 (2014)

13. Moudafi, A: A note on the split common fixed-point problem for quasi nonexpansive operators. Nonlinear Anal. 74, 4083-4087 (2011)

14. Kraikaew, R, Saejung, S: On split common fixed-point problems. J. Math. Anal. Appl. 415, 513-524 (2014)

15. Censor, Y, Gibali, A, Reich, S: Algorithms for the split variational inequality problem. Numer. Algorithms 59, 301-323 (2012)

16. Ansari, QH, Nimana, N, Petrot, N: Split hierarchical variational inequality problems and related problems. Fixed Point Theory Appl. 2014, Article ID 208 (2014)

17. Ansari, QH, Ceng, L-C, Gupta, H: Triple hierarchical variational inequalities. In: Ansari, QH (ed.) Nonlinear Analysis: Approximation Theory, Optimization and Applications, pp. 231-280. Springer, New York (2014)

18. Minty, GJ: On the generalization of a direct method of the calculus of variations. Bull. Am. Math. Soc. 73, 314-321 (1967)

19. Bruck, RE, Reich, S: Nonexpansive projections and resolvent of assertive operators in Banach spaces. Houst. J. Math. 3, 459-470 (1977)

20. Cegeilski, A: Iterative Methods for Fixed Point Problems in Hilbert Spaces. Springer, New York (2012)

21. Opial, Z: Weak convergence of the sequence of successive approximations for nonexpansive mappings. Bull. Am. Math. Soc. 73, 591-597 (1976)

22. Polyak, BT: Introduction to Optimization. Optimization Software Inc., New York (1987) 